\title{
PHOTOEMISSION ELECTRONIC STATES OF THALLIUM- AND BISMUTH-BASED SUPERCONDUCTORS
}

\author{
R. ZALECKI ${ }^{a}$, A. KolodzIEJCZYK ${ }^{a}$, J.W. KöNIG ${ }^{b}$ AND G. GritzneR ${ }^{b}$ \\ ${ }^{a}$ University of Mining and Metallurgy, Faculty of Physics and Nuclear Technique \\ Al. A. Mickiewicza 30, 30-059 Cracow, Poland \\ ${ }^{b}$ Johannes Kepler Universität, Institut für Chemische Technologie Anorganischer Stoffe \\ 4040 Linz, Austria
}

\begin{abstract}
X-ray photoemission spectra the core-levels as well as the $\mathrm{X}$-ray photoemission spectra and ultraviolet photoemission spectra from the valence bands of the $\left(\mathrm{Tl}_{0.6} \mathrm{~Pb}_{0.5}\right)\left(\mathrm{Sr}_{0.9} \mathrm{Ba}_{0.1}\right) \mathrm{Ca}_{2} \mathrm{Cu}_{3} \mathrm{O}_{y}$ and $\left(\mathrm{Bi}_{1.75} \mathrm{~Pb}_{0.35}\right) \mathrm{Sr}_{1.9} \mathrm{Ca}_{2.05} \mathrm{Cu}_{3.05} \mathrm{O}_{y}$ superconductors were measured and analyzed. Special attention was paid to the valence band X-ray photoemission spectra and ultraviolet photoemission spectra, the $\mathrm{Cu} 2 p$ core-level X-ray photoemission spectra and the $\mathrm{Cu} L_{2,3}-M_{4,5} M_{4,5}$ and $\mathrm{O} K-L_{2,3} L_{2,3}$ Auger spectra. Both $\mathrm{Cu} 2 p_{3 / 2}$ and $\mathrm{Cu} 2 p_{1 / 2}$ core-level lines consisted of two spin-orbit split main lines accompanied with the two satellite lines. The charge transfer energy $\Delta$ from the oxygen ligand to the copper $3 d^{9}$ states and the hopping integral $t$ were estimated from the energy separation between the main line and the satellite line taking advantage of the local cluster model calculations and their extension to high-temperature superconductors. The Coulomb correlation on-site energy $U_{d d}$ of two electrons in the same copper orbital and $U_{p p}$ of two electrons in the oxygen orbital as well as the correlation energy $U_{c d}$ of the $2 p$ core hole $-3 d$ electron interaction have been estimated from the Auger electron spectra and the valence band spectra. They are: $U_{d d}=6.0 \pm 0.5 \mathrm{eV}$, of $U_{p p} \cong 10 \pm 1 \mathrm{eV}$ and of $U_{c d} \cong 8.0 \pm 0.5 \mathrm{eV}$ nearly the same for both the Tl- and Bi-compounds. We conclude that these compounds are the charge transfer strongly-correlated metals.
\end{abstract}

PACS numbers: 74.72.-h, 79.60.-i

\section{Introduction}

The presence of electron correlations in high-temperature superconductors (HTS) is the one among others most important effect in these materials. Physical properties like magnetism in strongly correlated systems, heavy fermion behavior, giant magnetoresistance, etc. are governed by strong electron-electron interactions. The one - electron Bloch-like theory is insufficient to describe their physical 
properties. Solids are many-electron systems and the electrons interact with each other via the Coulomb and exchange interaction. To understand the nature of HTS it is important to have knowledge of their correlated electronic states. Photoelectron spectroscopy (PES) is one of the most important tool to probe these electronic states. The basic problem with the interpretation of the $d$-electron photoemission in HTS stems from the fact of large correlation effect among $3 d$ electrons and the hybridization of the metal $d$-electrons with the ligand $p$-electrons.

The theory of the electronic structure in the transition metal (TM) compounds was developed in [1-5]. The basic electronic features of TM compounds were described by the so-called Zaanen, Sawatzky and Allen phase diagram [2] $U_{d d} / t$ versus $\Delta / t$. $U_{d d}$ is the $d-d$ on-site electron (hole) Coulomb correlation energy (Hubbard energy), $\Delta$ is the charge transfer energy for the excitation of an electron from a ligand ion onto the $d$-hole state of metal ion, $t$ is the hybridization term between $p$ and $d$ states (effective hybridization term $T_{\text {eff }}$ ), sometime called the transfer integral, associated with the $p-d$ Coulomb correlation energy $U_{c d}$ between $2 p$ core hole and $3 d$ holes. This theory included the correlation and hybridization effects.

One would normally expect the $U_{d d}$ to increase and the bare charge-transfer $\Delta$ to decrease for the TM compounds with higher atomic numbers. As estimated from calculations [6] and electron-spectroscopy studies [7] the one-site Coulomb correlation energy is known to be large for the late TM and their compounds.

The dominating ground states in the investigated samples are one-hole states and the two-hole states are the minority. In the photoemission the local cluster model calculations were thoroughly used [8-13]. The model assumes in copper oxides the existence of separated $\left(\mathrm{CuO}_{6}\right)^{10-}$ cluster and it describes the copper $3 d$ electrons hybridized to the oxygen $2 p$ states. The ground states are assumed as a linear combination of the $d^{9}$ and $d^{10} L^{-1}$ configurations. The final states in the copper $2 p$ photoemission are the linear combination of $2 p^{5} 3 d^{9}$ and $2 p^{5} 3 d^{10} L^{-1}$ configurations. From this approach one can elaborate the photoemission spectra and estimate the $U_{c d}, \Delta$, and $t$ values. Especially, the satellite to main peak energy distance $\Delta E_{M-S}$ in the Cu $2 p$ core-level X-ray photoemission spectra (XPS) was expressed in $[8-10,14]$ as a function of the $U_{c d}, \Delta$, and $t$.

Also the structure of the $\mathrm{Cu} L_{2,3}-M_{4,5} M_{4,5}$ Auger line for copper compounds may be analyzed according to the model presented in [8]. In the present paper the quantitative estimates of the $U_{d d}$ as well as the $U_{p p}$, which is the on-site Coulomb correlation energy of two electrons (holes) in the oxygen orbital, were made from the relevant Auger lines and the "copper" and "oxygen" parts of the valence band.

For the high-temperature superconductors a lot of photoemission and Auger electron spectroscopic studies have been reported up to now [15-27]. Among them the most important resonant photoemission valence band spectra and the angle resolved photoemission in the binding energy region close to the Fermi surface have been carried out [14, 17-25].

It is already well-established that the high-temperature superconductivity appears due to doping by holes like in $\mathrm{YBa}_{2} \mathrm{Cu}_{3} \mathrm{O}_{7-\delta}$ or by electrons like in $\mathrm{Nd}_{2-x} \mathrm{Ce}_{x} \mathrm{CuO}_{4-y}$. In the former system the charge neutrality requires the con- 
version of $\mathrm{Cu}^{2+}$ to $\mathrm{Cu}^{3+}$ or the converting $\mathrm{O}^{-2}\left(2 \mathrm{p}^{6}\right)$ to $\mathrm{O}^{-1}\left(2 p^{5}\right)$. It means an appearance of holes $L^{-1}$ in the oxygen $2 p$ band $\left(L^{-1}\right.$ indicates an oxygen ligand hole state) yielding the formally trivalent copper $\mathrm{Cu}^{3+}$ instead of the real $\mathrm{Cu}^{3+}\left(3 d^{8}\right)$ states. It was proved that the superconductivity is determined by the formally trivalent copper $\mathrm{Cu}^{3+}$ with the $3 d^{9} L^{-1}$ electronic states, usually called formally trivalent copper because the extra electron missing with respect to divalent copper $\mathrm{Cu}^{2+}$ is taken from the oxygen $2 p$ reservoir rather than from the $\mathrm{Cu}$ $3 d$ reservoir [15-27].

The valence band (VB) states are a mixture of ligand $(L) p$-states and metal $d$-states and thus can be written as $d^{9} L$ states. This valence band leads to two final states after photoemission, namely $d^{8} L$ and $d^{9} L^{-1}$. The first state corresponds to the creation of a $d^{8} L$ state (e.g. $\mathrm{Cu}^{3+} 3 d^{8} 2 p^{6}$ ) out of $d^{9} L$ state (e.g. $\mathrm{Cu}^{2+} 3 d^{9} 2 p^{6}$ in $\mathrm{CuO}$ ) which requires a correlation energy $U_{d d}$. The second final state $d^{9} L^{-1}\left(3 d^{9} 2 p^{5}\right)$ is produced by a $p$-ligand to $d$-metal charge transfer out of the photoionized state $d^{8} L$ due to hybridization. It needs a charge transfer energy $\Delta$. Thus two types of electronic excitations are possible in VB of transition metal oxides. The first excitation of $d$-electron from TM ion and its transfer to another distant transition metal ion which are governed by the energy $U_{d d}$. The second excitation is the transfer of a ligand electron onto a transition metal ion described by the energy $\Delta$. The relative magnitude of these two energies $U_{d d} / \Delta$ defines whether a compound is a Mott insulator (MI) $\left(U_{d d}<\Delta\right)$ (or more generally, a Mott compound) or a charge-transfer compound (CTC) $\left(U_{d d}>\Delta\right)$.

In the present paper the XPS and ultraviolet photoemission spectra (UPS) of the $\left(\mathrm{Tl}_{0.6} \mathrm{~Pb}_{0.5}\right)\left(\mathrm{Sr}_{0.9} \mathrm{Ba}_{0.1}\right)_{2} \mathrm{Ca}_{2} \mathrm{Cu}_{3} \mathrm{O}_{y}$ and $\left(\mathrm{Bi}_{1.75} \mathrm{~Pb}_{0.35}\right) \mathrm{Sr}_{1.9} \mathrm{Ca}_{2.05} \mathrm{Cu}_{3.05} \mathrm{O}_{y}$ superconductors were measured and analyzed. Special attention was paid to the valence band XPS/UPS, the Cu $2 p$ core-level XPS and the $\mathrm{Cu} L_{2,3}-M_{4,5} M_{4,5}$ and $\mathrm{O} K-L_{2,3} L_{2,3}$ Auger spectra to estimate the all energies and parameters characterizing the electron correlation effects in a consistent way for the first time.

\section{Experimental}

Polycrystalline pellets of the $\left(\mathrm{Tl}_{0.6} \mathrm{~Pb}_{0.5}\right)\left(\mathrm{Sr}_{0.9} \mathrm{Ba}_{0.1}\right)_{2} \mathrm{Ca}_{2} \mathrm{Cu}_{3} \mathrm{O}_{y}$ and $\left(\mathrm{Bi}_{1.75} \mathrm{~Pb}_{0.35}\right) \mathrm{Sr}_{1.9} \mathrm{Ca}_{2.05} \mathrm{Cu}_{3.05} \mathrm{O}_{y}$ superconductors were prepared by solid-state reaction with the superconducting transition temperature $T_{\mathrm{c} 0}$ of $118 \mathrm{~K}$ and $103 \mathrm{~K}$, respectively, by a procedure described elsewhere [28-30].

We employed a system XPS/UPS (ultraviolet) Leybold LHS10 equipped with the dual Al- and $\mathrm{Mg}-K_{\alpha} \mathrm{X}$-ray sources with a typical resolution of $0.85 \mathrm{eV}$ and with the high intensity UV source with the energy resolution of about $50 \mathrm{meV}$. As a test of contamination - free surface the intensity of carbon $\mathrm{C} 1 \mathrm{~s}$ typical peak at about $298 \mathrm{eV}$ was used prior to experiment under the vacuum better than $10^{-9}$ Torr. The $\mathrm{C} 1 s$ signal due to carbonate impurities was negligible for all samples. The spectrometer was calibrated using the $\mathrm{Ag}$ Fermi edge, the $\mathrm{Ag} 3 d_{5 / 2}$ line and the $\mathrm{Cu} 2 p_{3 / 2}$ white line to which the binding energies $0 \mathrm{eV}, 368.3 \mathrm{eV}$, and $932.7 \mathrm{eV}$ were assigned, respectively. The experiments were performed also at the $\mathrm{LN}_{2}$ temperature for comparison and to avoid surface degradation. The cleanliness of sample surface was ensured by in situ scraping with a diamond file. 


\section{Results and their analysis}

The full energy range spectra in room temperature are shown in Fig. 1 with the indicated important photoemission lines.

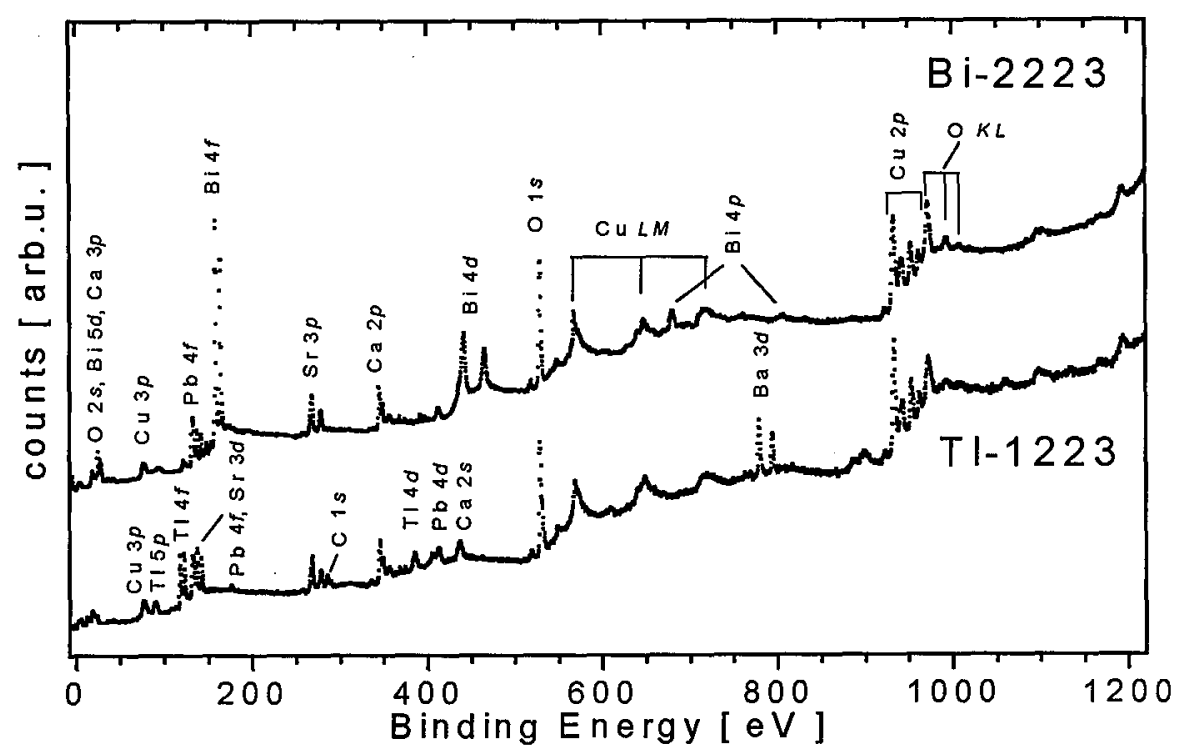

Fig. 1. The full scan XPS (Al- $\left.K_{\alpha}\right)$ of the $\left(\mathrm{Bi}_{1.75} \mathrm{~Pb}_{0.35}\right) \mathrm{Sr}_{1.9} \mathrm{Ca}_{2.05} \mathrm{Cu}_{3.05} \mathrm{O}_{y}(\mathrm{Bi}-2223)$ and $\left(\mathrm{Tl}_{0.6} \mathrm{~Pb}_{0.5}\right)\left(\mathrm{Sr}_{0.9} \mathrm{Ba}_{0.1}\right)_{2} \mathrm{Ca}_{2} \mathrm{Cu}_{3} \mathrm{O}_{y}$ ( $\left.\mathrm{Tl}-1223\right)$ superconductors with the important lines indicated.

Figure 2 shows the $\mathrm{Cu} 2 p_{3 / 2}$ and $2 p_{1 / 2}$ XPS (Al- $\left.K_{\alpha}, 1486.6 \mathrm{eV}\right)$ core-level spectra in room temperature. They exhibit the main lines $M$ at $932.5 \mathrm{eV}(J=3 / 2)$ and at $952.5 \mathrm{eV}(J=1 / 2)$, respectively.

In the $\mathrm{Cu} 2 p$ core-level spectra the broad satellites $S_{1}$ and $S_{2}$ appear at the $941.9 \mathrm{eV}$ and at $961.6 \mathrm{eV}$ binding energy, respectively, in addition to the spin-orbit split main lines $M_{1}$ and $M_{2}$ sometime with the additional structure. The satellites are characteristic of other insulating cuprates $[14,32]$ with $\mathrm{Cu}^{2+}$ ions with $S=1 / 2$ spins. The satellite results from the multiplet splitting effects due to the interaction between the $2 p$ core hole and the $3 d^{9}$ electronic configuration in the final state of the photoemission process $[31,32]$. In the core $\mathrm{Cu} 2 p$ spectrum the energy difference between the main $M_{1}$ and satellite $S_{1}$ peak is about $1 \mathrm{eV}$ larger than in other insulating cuprates $[14,32]$ which means a larger charge transfer energy $t$ between $2 p$ and $3 d$ holes. It was established that the $M$ line occurs for significant different energy for different compounds while the satellite has roughly the same binding energy in all compounds [14]. The final state configurations of the main and satellite lines are labelled in Fig. 2.

Generally speaking, in the ground state the copper oxides with the $\mathrm{Cu}^{2+}$ ions have one hole in the $d$-shell ( $3 d^{9}$ configuration) and a filled ligand shell $L$. 


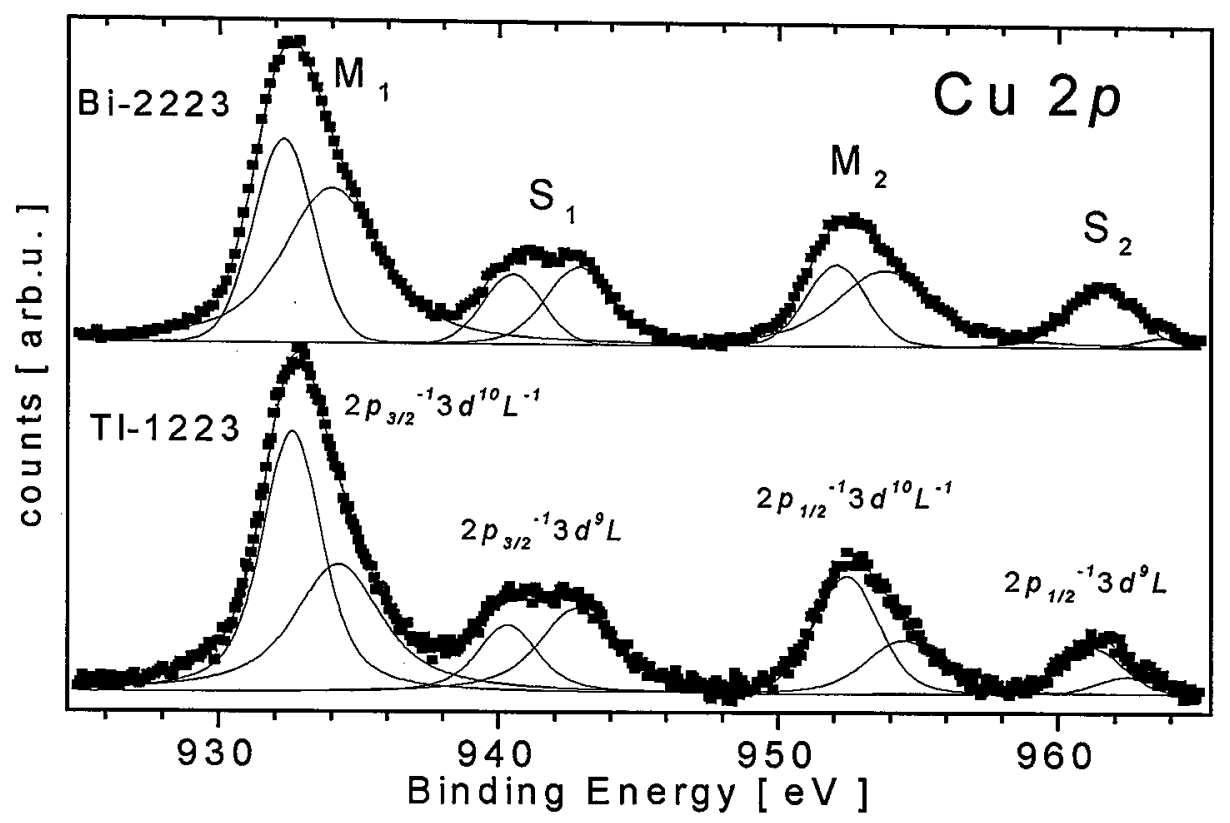

Fig. 2. The $\mathrm{Cu} 2 p$ core-level spectra of the $\left(\mathrm{Bi}_{1.75} \mathrm{~Pb}_{0.35}\right) \mathrm{Sr}_{1.9} \mathrm{Ca}_{2.05} \mathrm{Cu}_{3.05} \mathrm{O}_{y}$ (Bi-2223) and $\left(\mathrm{Tl}_{0.6} \mathrm{~Pb}_{0.5}\right)\left(\mathrm{Sr}_{0.9} \mathrm{Ba}_{0.1}\right)_{2} \mathrm{Ca}_{2} \mathrm{Cu}_{3} \mathrm{O}_{y}$ (Tl-1223) superconductors after subtraction of the background, with the best fitted lines (solid lines) and with the final state configurations as indicated.

The notation is $3 d^{9} L$ and refers to the $\mathrm{Cu}^{2+}$ ion and $L$ represents the fully occupied oxygen $2 p$ shell ( $2 p^{6}$ configuration). The photoexcitation in the $\mathrm{Cu} 2 p$ shell leads to two final states: one, where after creation of the $2 p$ core hole the ground state configuration $3 d^{9} \mathrm{~L}$ is left roughly intact; another one, where one electron is transferred from the ligand shell $L$ into the $d$-shell due to relaxation process, leading to a $3 d^{10} L^{-1}$ configuration. The latter configuration applies to the $M$ line and hence the binding energy is: $E_{M}=E\left(c^{-1} d^{10} L^{-1}\right) \cong E\left(c^{-1} d^{10}\right)+E\left(L^{-1}\right)$ where $c^{-1}$ indicates core hole in $\mathrm{Cu} 2 p$ shell. By the same reasoning the $S$ line is described by energy $E_{S}=E\left(c^{-1} d^{9} L\right)$. Thus, the main line energy is different because is influenced by a strong correlation with the ligand in the sense that the production of a hole in the ligand valence band orbital depends on the ligand ion. This is not the case for $S$ line. In the ground state of TM compounds the $3 d$ valence shell is usually in energy above the ligand valence orbitals. However, the additional Coulomb interaction $U_{c d}$ may pull it below the top of the ligand valence band. This is the case in HTS. This is an unstable situation which does not correspond to the ground state but to the satellite. It is then energetically possible to transfer an electron from the ligand band into the empty $3 d$ orbital and the ion attains its ground state. In such a case the initial state configurations for $\mathrm{Cu}$ are $3 d^{9} L$ and $3 d^{10} L^{-1}$ with some mixing. These configurations correspond to two states of which one is filled (mostly $3 d^{9} L$ ) and the other is empty (mostly $3 d^{10} L^{-1}$ ) in the initial state. These two states can be populated in the final state creating a 
main $M$ line (mostly $3 d^{10} L^{-1}$ ) and a satellite $S$ line (mostly $3 d^{9} L$ ). That is why the fits were made with two lines as shown in Fig. 2 subtracting the background according to the Tougaard procedure [33]. The combination of the Lorentzian and Gauss line was fitted to the experimental data of the $\mathrm{Cu} 2 p$ core level spectra.

Figure 3 shows the room and liquid nitrogen temperature XP and UP spectra of the valence bands of both superconductors. The spectra are very similar for both superconductors with exception that the Fermi edge $E_{\mathrm{F}}$ for policrystalline material is only observed for Bi-2223 specimen. The edge is a very good zero reference point for calibration of energy scale in further analysis of the photoemission spectra. It was already established that the $\mathrm{O} 2 p$ states participate in VB mostly close to the Fermi level whereas the $\mathrm{Cu} 3 d$ states are contributing to the bottom of VB [14-19]. Therefore, the position of the leading $2 p$ emission is at $-2.7 \mathrm{eV}$ below $E_{\mathrm{F}}$ and of the $3 d$ emission is at $-5.1 \mathrm{eV}$ below $E_{\mathrm{F}}$ as roughly estimated with two lines fitting shown in Fig. 3. These energy values will be used to analyze the relevant Auger lines together with the VB spectrum of Bi-2223 in the next paragraph.

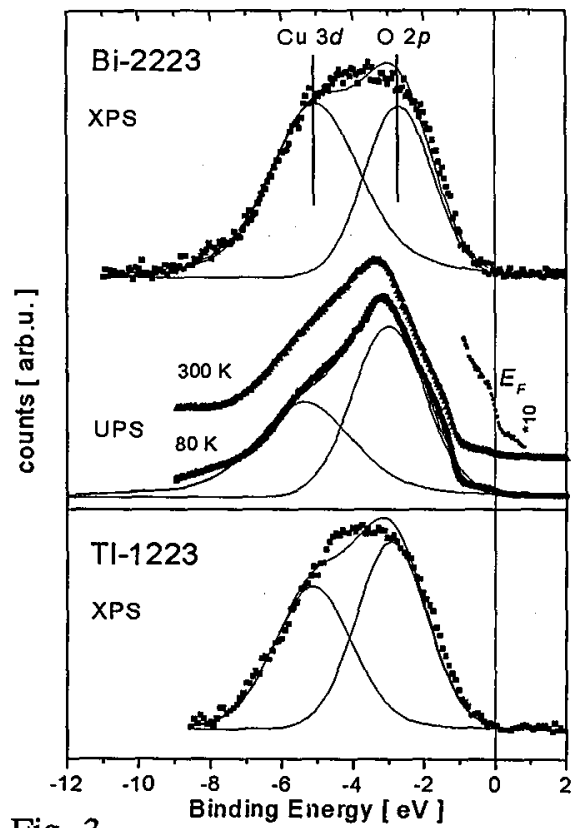

Fig. 3

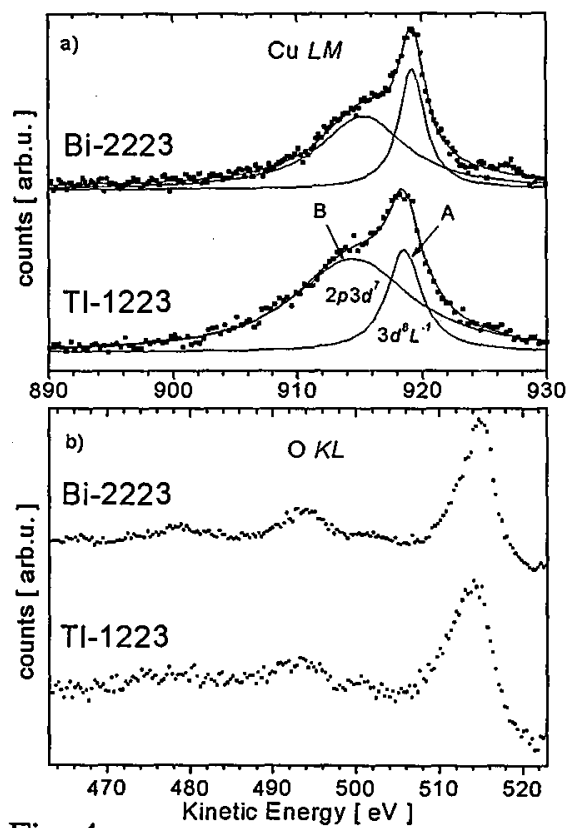

Fig. 4

Fig. 3. The X-ray photoemission ( $\left.\mathrm{Mg}-K_{\alpha}\right)$ and ultra violet photoemission (He-I) spectra. The $\mathrm{Cu} 3 d$ and $\mathrm{O} 2 p$ emissions together with the Fermi edge in Bi-2223 UPS spectra are shown.

Fig. 4. Auger electron lines (a) of Cu: $L_{3}-M_{4,5} M_{4,5}$ and (b) of O: $K-L_{2,3} L_{2,3}$ with the final state configurations for copper as indicated.

The $\mathrm{Cu} L_{2,3}-M_{4,5} M_{4,5}$ Auger lines of $M_{4,5} M_{4,5}$ and of $\mathrm{O} K-L_{2,3} L_{2,3}$ in room temperature are shown in Fig. 4 . The $\mathrm{Cu} L_{2,3}-M_{4,5} M_{4,5}$ Auger line for both superconductors consists of the main high-kinetic energy component $(A)$ at 
$E_{A}=919.2 \mathrm{eV}$ and the second lower-kinetic energy component $(B)$ at $E_{B}=$ $915.3 \mathrm{eV}$ as estimated after fitting two lines to the measured spectrum and after subtraction of the background according to the Tougaard procedure [33]. The line $A$ was attributed to the $3 d^{8} L^{-1}$ final state configuration after the Auger process starting from a $2 p_{3 / 2} 3 d^{10} \mathrm{~L}^{-1}$ intermediate state. The line $B$ was assigned to the $3 d^{7}$ final configuration from a $2 p_{3 / 2} 3 d^{9}$ intermediate state [8].

The energy positions of the XPS Cu $2 p$ lines, of the Auger lines and of the $\mathrm{Cu} 3 d$ and $\mathrm{O} 2 p$ emission form XPS valence bands as taken from the experimental spectra and from the fitting procedures are collected in the Table.

TABLE

The experimental and fitted energy positions $[\mathrm{eV}]$ of the $\mathrm{Cu} 2 p$ and Auger lines together with the valence band energy positions of the $\mathrm{Cu} 3 d$ and $\mathrm{O} 2 p$ emissions. (The estimated maximal error of the values given in the Table is about $0.2 \mathrm{eV}$.)

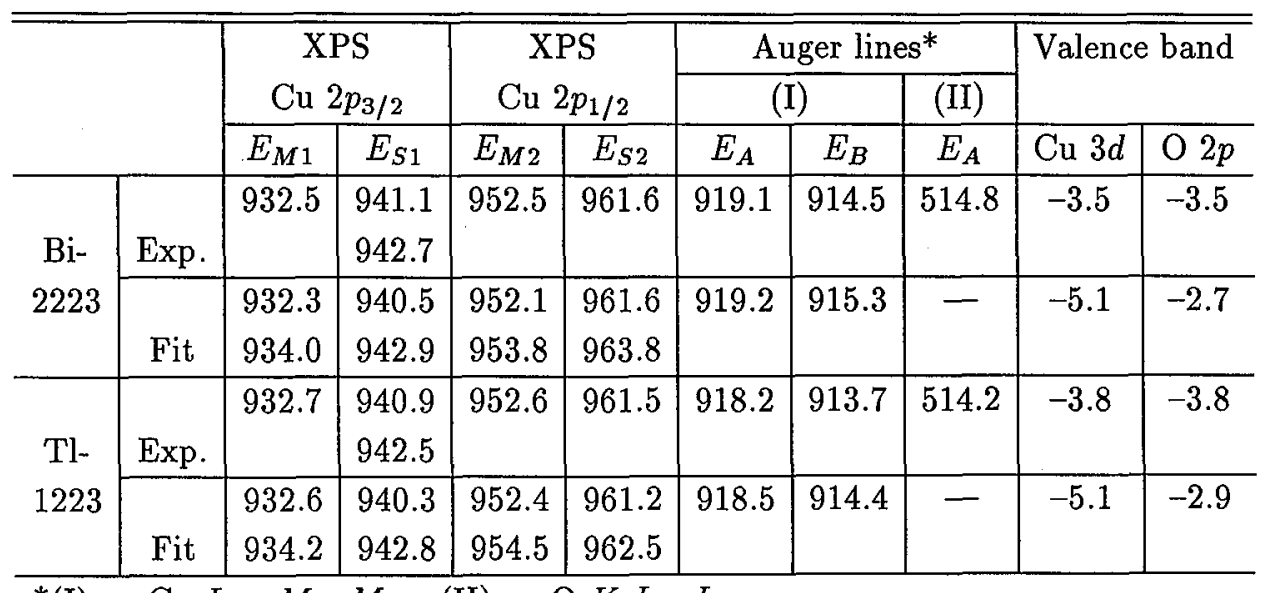

*(I) $-\mathrm{Cu} L_{2,3}-M_{4,5} M_{4,5}$, (II) $-\mathrm{O} K-L_{2,3} L_{2,3}$

\subsection{Analysis of Auger lines together with the valence band}

In order to estimate the Coulomb correlation strength $U_{d d}$ and $U_{p p}$ one can use Auger electron lines of $\mathrm{Cu} L_{3}-M_{4,5} M_{4,5}$ at about $919 \mathrm{eV}$ and of $\mathrm{O} K-L_{2,3} L_{2,3}$ at about $515 \mathrm{eV}$ shown in Fig. 4. The spectral features do not significantly change for both superconductors indicating very similar Coulomb correlation energies, $U_{d d}$ as well as $U_{p p}$, in these compounds. In order to extract a quantitative estimate of $U_{d d}$, we have compared the $\mathrm{Cu} L_{2,3}-M_{4,5} M_{4,5}$ Auger spectrum with the self-convoluted density of states (DOS) after aligning them to the respective Fermi energies $E_{\mathrm{F}}=0$. To do this we have followed the procedure presented in [34]. The position of relevant Auger line was compared with the self-convoluted DOS of the XP spectra of the valence band taking into account that the $\mathrm{O} 2 p$ states participate in VB mostly close to the Fermi level $E_{\mathrm{F}}$ whereas the $\mathrm{Cu} 3 d$ states are contributing to the bottom of VB (see Fig. 3). The peak at $-2.7 \mathrm{eV}$ below $E_{\mathrm{F}}$ is primarily due 
to the oxygen $2 p$ emission and the peak at $-5.1 \mathrm{eV}$ below $E_{\mathrm{F}}$ comes mainly from the copper $3 d$ emission [14-29]. The procedure is shown in Fig. 5.

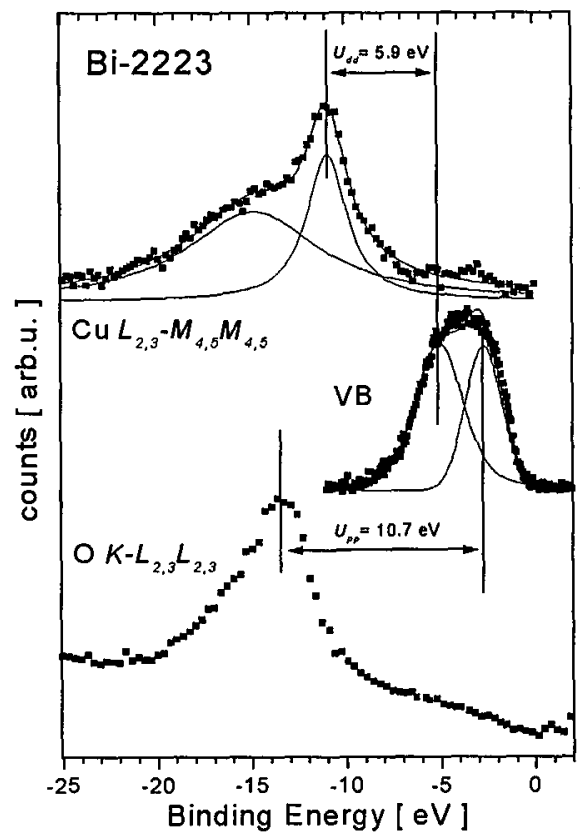

Fig. 5. The valence band $\mathrm{Cu} 3 d$ and $\mathrm{O} 2 p$ emissions along with the $\mathrm{Cu}: L_{3}-M_{4,5} M_{4,5}$ and of $\mathrm{O} K-L_{2,3} L_{2,3}$ Auger spectra with their respective Fermi energies aligned and the $U_{d d}$ and $U_{p p}$ energies as indicated.

The energy difference between the valence band copper emission and the high energy peak $A$ in the $\mathrm{Cu} L_{2,3}-M_{4,5} M_{4,5}$ Auger spectrum provides us with an estimate of $U_{d d} \cong 5.9 \pm 0.5 \mathrm{eV}$. The energy difference between the valence band oxygen emission and the $\mathrm{O} K-L_{2,3} L_{2,3}$ Auger line gives an estimate of $U_{p p} \cong 10.4 \pm 1 \mathrm{eV}$. We can use here an additional relation $U_{c d} \cong 1.4 U_{d d}$ which is consistent with a large body of experimental results. From this we have $U_{c d}=$ $8.3 \pm 0.5 \mathrm{eV}$. Finally, we can compare the relevant copper/oxygen VB emission with oxygen/copper Auger line, respectively. This procedure gives an estimate $U_{c d}=U_{d c}=8.3 \pm 1 \mathrm{eV}$ which is the same as the previous one. The error is due to the accuracy of the alignment of the relevant Auger lines to the Fermi energy.

Also the structure of the $\mathrm{Cu} L_{2,3}-M_{4,5} M_{4,5}$ Auger line for copper compounds may be analyzed according to the model presented in [8]. The main peak $A$ is due to a correlated two-hole final state and the shoulder $B$ is due to transitions into the uncorrelated two-hole final state. The line $A$ was attributed to the $3 d^{8} L^{-1}$ final state configuration after the Auger process starting from a $2 p_{3 / 2} 3 d^{10} L^{-1}$ intermediate state. The line $B$ was assigned to the $3 d^{7}$ final configuration from a $2 p_{3 / 2} 3 d^{9}$ intermediate state [8]. It was calculated [8] that

$$
E_{A}-E_{B} \cong 2 U_{d d}-U_{c d}
$$


and this relation was used in the estimation of $U_{c d}$. Having the value of $U_{d d}$ one can calculate the value of $U_{c d}$ taking into account the above relation and the experimental value of $E_{A}-E_{B} \cong 4.0 \mathrm{eV} \pm 0.3 \mathrm{eV}$ (c.f. the Table and Fig. 4). Hence $U_{c d}=7.9 \pm 0.5 \mathrm{eV}$.

The estimations of $U_{d d}, U_{p p}$, and $U_{c d}$ are in agreement with the estimates of the same quantities of other copper oxide superconductors and strongly correlated copper compounds [14, 32].

\subsection{Analysis of charge transfer satellites}

As it was said the two possible VB excitations are possible. The first $d-d$ excitation needs the energy $U_{d d}=E\left(d^{n+1}\right)+E\left(d^{n-1}\right)-2 E\left(d^{n}\right)$. The second excitation from $p$-ligand ion (configuration $L$ ) onto the $d^{n}$ configuration of a metal ion needs the energy $\Delta=E\left(d^{n+1}\right)+E\left(L^{-1}\right)-E\left(d^{n}\right)-E(L)$. Creation of a core hole shifts the $d^{n+1} L^{-1}$ but not $d^{n} L$ configuration down by an amount $U_{c d}$ so that the difference between the satellite line and the main line is $\Delta E_{M-S}=\left(U_{c d}-\Delta\right)$ depending on the relative magnitude of $U_{c d}$ and $\Delta$. If one takes into consideration the hybridization effect between the metal ions and the ligand ions a more general description which is applicable to any $d^{n}$ configuration was elaborated in [8-10]. From this approach one can elaborate the photoemission spectra. Especially, the satellite to the main peak energy distance in the Cu $2 p$ core-level XP spectra is expressed as [8]

$$
\Delta E_{M-S} \cong \sqrt{\left(U_{c d}-\Delta\right)^{2}+4 T_{m}^{2}}=\sqrt{\left(U_{c d}-\Delta\right)^{2}+4 t^{2}},
$$

where the mixing matrix element between the two initial states $\Delta=E\left(3 d^{10} L^{-1}\right)-$ $E\left(3 d^{9}\right)$ is given by $T_{m}=\left\langle 3 d^{9}|H| 3 d^{10} L^{-1}\right\rangle \cong t$.

For the analysis of the experimental satellite data according to the above formulae one needs other quantitative estimates of two quantities out of $\Delta, U_{c d}$, and $t$. Now we can use the value of $U_{c d}=8.3 \mathrm{eV}$ which has been just estimated in the previous paragraph. In addition according to the previous paragraph $\Delta E_{A-B} \cong 2 U_{d d}-U_{c d}=4.5 \pm 0.5 \mathrm{eV}$ from the Auger spectra just analyzed above. Hence, taking the value of $U_{d d}=5.9 \pm 0.5 \mathrm{eV}$ from the previous paragraph we have for $U_{c d}=7.9 \pm 0.5 \mathrm{eV}$ as an additional estimate. The relative size of $\Delta$ and $U_{c d}$ determines whether in a TM compound with configuration $d^{n} L$ in the initial state after photoionization the same $d^{n} L$ configuration is the lowest final state or whether the $d^{n+1} L^{-1}$ configuration is the final state. This model forms the basis for analysis photoemission data. Hence, taking into account the above formulae one can estimate the values of $t$ assuming that $\Delta$ is about $1.5 \mathrm{eV}$ which is again consistent with a large number of experimental results and theoretical calculations [14-34]. Thus a complete set of parameters is

$$
\begin{aligned}
& U_{d d}=5.9 \pm 0.5 \mathrm{eV}, \quad U_{c d}=7.9 \pm 0.5 \mathrm{eV}, \\
& U_{p p} \cong 10.4 \pm 1 \mathrm{eV} \text { and } t=1.3 \pm 0.5 \mathrm{eV},
\end{aligned}
$$

for $\Delta=1.5 \mathrm{eV}$. From these values the investigated superconductors must be classified as the charge transfer strongly-correlated hole-type metals according to Zaanen-Sawatzky-Allen (ZSA) phase diagram [2]. An additional argument for the charge transfer behavior of these compounds is the majority contribution from the oxygen $2 p$ emission to the top of valence band (c.f. Fig. 3). 


\section{Summary and conclusions}

1. Our XPS, UPS, and Auger spectra of $\left(\mathrm{Tl}_{0.6} \mathrm{~Pb}_{0.5}\right)\left(\mathrm{Sr}_{0.9} \mathrm{Ba}_{0.1}\right)_{2} \mathrm{Ca}_{2} \mathrm{Cu}_{3} \mathrm{O}_{y}$ and $\left(\mathrm{Bi}_{1.75} \mathrm{~Pb}_{0.35}\right) \mathrm{Sr}_{1.9} \mathrm{Ca}_{2.05} \mathrm{Cu}_{3.05} \mathrm{O}_{y}$ superconductors were assigned to the photoemission electronic final state configurations according to the standard approach for copper compound and the great number of spectroscopic results collected up to now. We have observed the Fermi edge in the valence band of the bismuth superconductor which was a very good reference energy for analysis of the recorded spectra.

2. From the energy difference between the $\mathrm{Cu}$ valence band emission and the high energy peak $A$ of the $\mathrm{Cu} L_{2,3}-M_{4,5} M_{4,5}$ Auger spectrum the on-site $d-d$ Coulomb correlation energy was estimated to be $U_{d d} \cong 5.9 \pm 0.5 \mathrm{eV}$. The energy difference between the oxygen valence band emission and the O $K-L_{2,3} L_{2,3}$ Auger line gives an estimate of the on-site $p-p$ Coulomb correlation energy $U_{p p} \cong 10.4 \pm 1 \mathrm{eV}$. From the energy separation between the two components of the $\mathrm{Cu} L_{2,3}-M_{4,5} M_{4,5}$ Auger line the $2 p$ core hole - $3 d$ hole correlation energy was calculated to be $U_{c d}=7.9 \pm 0.5 \mathrm{eV}$.

3. The hoping integral $t=1.3 \pm 0.5 \mathrm{eV}$ was extracted from the satellite to the main peak energy distance in the $\mathrm{Cu} 2 p$ core-level XP spectra and assuming the charge transfer energy to be $\Delta=1.5 \mathrm{eV}$.

4. These correlation energies and the relative magnitude of $U_{d d}>\Delta$ define that both superconductors are the charge transfer strongly-correlated hole-type metals according to the phase diagram $U_{d d} / t$ versus $\Delta / t[2]$.

\section{Acknowledgments}

This study was financially supported by the Committee for Scientific Research (Poland) (project No. 2P03B 147 17), and by the Österreichischer Akademischer Austauschdienst, Wissenschaftlich-technische Zusammenarbeit mit Polen (project 3/99). We thank Prof. A. Szytuła and Mr. B. Penc for making possible the photoemission experiments in the Institute of Physics of the Jagiellonian University in Cracow.

\section{References}

[1] N.F. Mott, J.H. Davies, Electronic Processes in Non-Crystalline Materials, Oxford University Press, Oxford 1979, p. 197.

[2] J. Zaanen, G.A. Sawatzky, J.W. Allen, Phys. Rev. Lett. 55, 418 (1985).

[3] G.A. Sawatzky, J.W. Allen, Phys. Rev. Lett. 53, 2339 (1984).

[4] J. Zaanen, G.A. Sawatzky, J. Solid State Chem. 88, 8 (1990).

[5] H. Eskes, G.A. Sawatzky, Phys. Rev. B 43, 119 (1991).

[6] T. Bandyopadhyay, D.D. Sarma, Phys. Rev. B 39, 3517 (1989).

[7] A. Fujimori, F. Minami, S. Sugano, Phys. Rev. B 29, 5225 (1984). 
[8] G. van der Laan, C. Westra, C. Haas, G.A. Sawatzky, Phys. Rev. B 23, 4369 (1981).

[9] S. Asada, S. Sugano, J. Phys. Soc. Jpn. 41, 1291 (1976).

[10] M.A. van Veenendaal, G.A. Sawatzky, Phys. Rev. B 49, 3473 (1994).

[11] Z.-X. Shen, J.W. Allen, J.J. Yeh, J.-S. Kang, W. Ellis, W. Spicer, I. Landau, M.B. Maple, Y.D. Dalichaouch, M.S. Torikachvili, J.Z. Sun, T.H. Geballe, Phys. Rev. $B$ 36, 8414 (1987).

[12] J. Ghijsen, G.A. Sawatzky, M.T. Czyzyk, Phys. Rev. B 38, 11322 (1988).

[13] G. Chiaia, M.Qvarford, I. Lindau, S. Söderholm, U.O. Karlsson, S.A. Flodström, L. Leonyuk, A: Nilsson, N. Mårtensson, Phys. Rev. B 51, 1213 (1995).

[14] S. Hüfner, Photoelectron Spectroscopy, in Springer Series in Solid-State Sciences, Vol. 82, Springer-Verlag, Berlin 1995, p. 226 and references cited therein.

[15] for early review see A. Bianconi, in: Proc. the Taniguchi Int. Symposium on Core Level Spectroscopy in Condensed System, Eds. A. Kotani, J. Kanamori, Springer Verlag, Berlin 1989, p. 99.

[16] A. Kołodziejczyk, Mol. Phys. Report 15/16, 11 (1996) and references cited therein.

[17] P. Steiner, S. Hüfner, V. Kinsinger, I. Sander, B. Siegwart, H. Schmitt, R. Schulz, S. Junk, G. Schwitzgebel, A. Gold, C. Politis, H.P. Müller, R. Hoppe, S. Kemmler-Sack, C. Kunz, Z. Phys. B69, 449 (1988).

[18] For review see Fermiology of High- $T_{c}$ Superconductors, Eds. A. Bansil, A.J. Arko, R. Benedek, V.J. Emery, L.C. Smedskjaer, J. Phys. Chem. Solids 52, (1991).

[19] For review see Electronic Structure and Fermiology of High- $T_{c}$ Superconductors, Eds. T. Takahashi, A. Bansil, H. Katayama-Yoshida, J. Phys. Chem. Solids 53, (1992).

[20] J.C. Campuzano, G. Jennings, M. Faiz, L. Beaulaigue, B.W. Veal, J.Z. Liu, A.P. Paulikas, K. Vandervoort, H. Claus, R.S. List, A.J. Arko, R.J. Barlett, Phys. Rev. Lett. 64, 2308 (1990).

[21] R. Manzke, G. Mante, R. Claessen, M. Skibowski, J. Fink, Surf. Sci. 269, 1066 (1992).

[22] R. Liu, B.W. Veal, A.P. Paulikas, J.W. Downey, H. Shi, C.G. Olson, C. Gu, A.J. Arko, J.J. Joyce, Phys. Rev. B 45, 5614 (1992).

[23] J.G. Tobin, C.G. Olson, C. Gu, J.Z. Liu, F.R. Solal, M.J. Fluss, R.H. Howell, J.C. O'Brien, H.B. Radousky, P.A. Sterne, Phys. Rev. B 45, 5563 (1992).

[24] J.C. Campuzano, K. Gofron, R. Liu, H. Ding, B.W. Veal, B. Dabrowski, J. Phys. Chem. Solids 53, 1577 (1992).

[25] A.A. Abrikosov, J.C. Campuzano, K. Gofron, Physica C 214, 73 (1993).

[26] D.S. Dessau, Z.-X. Shen, B.O. Wells, W.E. Spicer, A.J. Arko, J. Phys. Chem. Solids 52, 1401 (1992).

[27] R.O. Anderson, R. Claessen, J.W. Allen, C.G. Olson, C. Janowitz, L.Z. Liu, H.-H. Park, M.B. Maple, Y. Dalichaouch, M.C. de Andrade, R.F. Jarodim, E.A. Early, S.-J. Oh, W.P. Ellis, Phys. Rev. Lett. 62, 336 (1988); Phys. Rev. Lett. 70, 3163 (1993).

[28] W. König, G. Gritzner, M. Reissner, W. Steiner, Physica C 258, 175 (1996).

[29] M. Mair, W. König, G. Gritzner, Supercond. Sci. Technol. 8, 894 (1995). 
[30] W. König, G. Gritzner, P. Diko, J. Kovác, M. Timko, J. Mater. Chem. 5, 879 (1995); W. König, M. Mair, G. Gritzner, J. Supercond. 11, 107 (1998).

[31] Z.-X. Shen, D.S. Dessau, Phys. Rep. 253, 1 (1995).

[32] R. Zimmermann, Ph.D. Thesis, Universität des Saarlandes, Saarbrücken 1996.

[33] S. Tougaard, Surf. Sci. 216, 343 (1989).

[34] A. Chainani, M. Mathew, D.D. Sarma, Phys. Rev. B 46, 9976 (1992). 